\title{
La Radioactivité
}

\author{
Sarah BAATOUT ${ }^{1}$ \\ Centre d'Étude de l'Énergie Nucléaire \\ SCK-CEN \\ Mol, Belgique.
}

\section{$\underline{\text { I. Définition de la radioactivité }}$}

La radioactivité est un phénomène physique naturel durant lequel des noyaux atomiques instables (radio-isotopes) se transforment spontanément (par désintégration) en dégageant de l'énergie sous forme de rayonnement pour se transformer en des noyaux atomiques plus stables, ayant perdu une certaine partie de leur masse. Autrement dit, c'est l'émission d'une particule ou d'un rayonnement par un noyau atomique.

Dans l'exemple ci-dessous, l'atome instable de radium-226 se désintègre et se transforme en un autre atome, le radium 222, tout aussi instable, en émettant un rayonnement alpha, provenant d'un noyau d'hélium. Le gros noyau lourd de radium-226 contient 226 nucléons, dont 88 protons et 138 neutrons. Il émet une particule alpha (noyau d'hélium) composée de deux protons et deux neutrons. Il se transforme alors en noyau de radon-222, lui-même radioactif, contenant deux protons et deux neutrons de moins. La désintégration libère 4,6 millions d'électronvolts d'énergie.

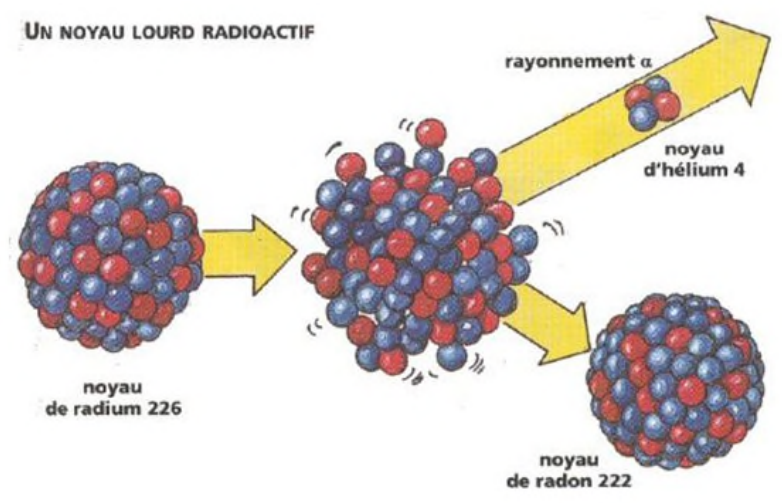

L'émission d'une particule alpha concerne surtout les très gros noyaux, le plus gros observé dans la nature étant celui de l'uranium-238 (qui comporte 92 protons et 136 neutrons).

De tels noyaux, instables, émettent un noyau léger d'hélium (particule alpha) afin de devenir moins volumineux et de se rapprocher ainsi de la stabilité. Cette manière d'expulser deux protons

\footnotetext{
1 e-mail : sbaatout@sckcen.be
}

Tél : +3214332729 
et deux neutrons groupés s'avère plus économique que d'expulser des protons et des neutrons de manière isolée.

Les noyaux radioactifs émettent trois sortes de rayonnements appelés par les physiciens alpha, bêta et gamma. Le fait d'appeler rayons alpha, bêta et gamma les noyaux d'hélium, les électrons (et positrons) ou les photons émis lors de désintégrations radioactives remonte à l'époque de la découverte car les premiers physiciens étaient férus de latin.

Sont nommés noyaux radioactifs «naturels », les noyaux légués par la nature dans notre environnement.

Le nombre de noyaux naturels est de 287. Par ailleurs, on dénombre plus de 2800 noyaux «artificiels », ce nombre continue de s'accroître avec les expérimentations et découvertes scientifiques diverses qui sont fabriqués en laboratoire. Certains noyaux, ont une durée de vie si courte qu'ils n'ont pas le temps de s'entourer d'un cortège électronique et de mériter le nom d'atome.

Certains radionucléides naturels ainsi que leur période et le type de rayonnements sont représentés dans le tableau ci-dessous.

\begin{tabular}{|l||c||l||c||}
\hline & Période & Unité & Emetteur \\
\hline \hline Uranium-238 & 4,468 & Milliards d'années & alpha \\
\hline \hline Thorium-234 & 24,10 & jours & bêta - \\
\hline \hline Protactinium-234 & 6,70 & heures & bêta - \\
\hline \hline Uranium-234 & 245500 & ans & alpha \\
\hline \hline Thorium-230 & 75380 & ans & alpha \\
\hline \hline Radium-226 & 1600 & ans & alpha \\
\hline \hline Radon-222 & 3,8235 & jours & alpha \\
\hline \hline Polonium-218 & 3,10 & minutes & alpha \\
\hline \hline Plomb-214 & 26,8 & minutes & bêta - \\
\hline \hline Bismuth-214 & 19,9 & minutes & bêta - \\
\hline \hline Polonium-214 & 164,3 & microsecondes & alpha \\
\hline \hline Plomb-210 & 22,3 & ans & bêta \\
\hline \hline Bismuth-210 & 5,015 & jours & bêta \\
\hline \hline Polonium-210 & 138,376 & jours & alpha \\
\hline \hline Plomb-206 & Stable & & \\
\hline \hline
\end{tabular}

\section{Les radioisotopes utilisés en médicine nucléaire :}

Les radioisotopes sont très utilisés dans divers diagnostics ou dans la recherche, en général. En médecine, les radioisotopes introduits ou injectés dans le corps du patient, émettent des rayonnements qui, après détection et traitement des résultats, vont permettre de fournir des 
informations sur l'anatomie de la personne ainsi que sur le fonctionnement de certains organes spécifiques. Ainsi utilisés, les radioisotopes portent le nom de traceurs.

La radiothérapie bénéficie également de certains radioisotopes, très utiles pour le traitement de certaines pathologies, comme le cancer, par exemple.

Environ $90 \%$ des radioisotopes sont utilisés à des fins diagnostiques et $10 \%$ pour de la thérapie. Enfin, afin de stériliser le matériel médical et de désactiver et tuer les bactéries éventuelles, des sources puissantes de rayons gamma sont utilisées. La stérilisation est quasi utilisée dans tous les pays du monde.

Par ailleurs, il est reconnu que dans les pays occidentaux, environ une personne sur deux bénéficiera de la médecine nucléaire au cours de sa vie.

Les radioisotopes peuvent être produits soit avec des cyclotrons, soit avec des réacteurs. Les grands hôpitaux sont en général équipés de cyclotrons, ce qui permet une utilisation immédiate. La production des radioisotopes, est obtenue par le bombardement d'une cible adaptée par des particules chargées, généralement des protons.

Les réacteurs nucléaires, comme le Belgian Reactor 2 (BR2), à Mol en Belgique, produisent la quasi-totalité des radio-isotopes utilisés à des fins thérapeutiques ainsi qu'une grande partie de ceux utilisés en imagerie médicale. Les réacteurs permettent la production de radioisotopes, non seulement, en plus grande quantité, mais également avec un coût plus faible. On utilise le bombardement d'une cible par des neutrons qui provoquent des réactions de fission. Les radioisotopes produits doivent cependant avoir une période radioactive assez longue pour permettre leur transport.

À noter que les réacteurs nucléaires assurent la production du molybdène-99 qui est le précurseur du technétium-99. Le technétium-99 est le principal radioisotope utilisé pour les radiodiagnostics (près de $75 \%$ des examens de scintigraphie). Le précurseur de ce radioisotope - le molybdène-99 - est fabriqué grâce à la fission en réacteur d'uranium -235. Environ 6\% des fissions de ce noyau fissile aboutissent à ce molybdène. Les cibles sont constituées de petites plaque d'aluminium contenant de l'uranium très enrichi en uranium-235.

\section{Les radioisotopes utilisés dans l'industrie :}

Les radioisotopes sont également utilisés pour étudier la fatigue des métaux, analyser des matériaux ou minéraux, examiner les soudures ou détecter les fuites. Ils sont aussi utilisés pour suivre et analyser les polluants, étudier les mouvements des eaux de surface, mesurer l'écoulement de la pluie et de la neige, ainsi que le débit des cours d'eau.

\section{La radiobiologie}

La radiobiologie est l'étude des effets biologiques des rayonnements, notamment des rayonnements ionisants, sur les êtres vivants. La recherche radiobiologique multidisciplinaire forme la base scientifique de la radioprotection, la radiothérapie et la médecine nucléaire. De ce fait, elle contribue à la santé publique. Dans le domaine de la radioprotection, son but est de 
mieux comprendre les effets d'une exposition aux radiations aux niveaux cellulaire et moléculaire afin de préciser leur impact sur la santé.

La radiobiologie est aussi l'étude des moyens de se protéger des effets délétères de certains rayonnements et l'étude des traitements à effectuer en cas de contamination radioactive et/ou irradiation. La radiobiologie actuelle bénéficie d'innombrables découvertes en cinétique cellulaire et en biologie moléculaire. Elle joue un rôle important dans l'évolution de la radiopathologie, la radiothérapie (externe ou ciblée) et de la radioprotection.

\section{La radiobiologie au SCK-CEN :}

Le propos de la recherche réalisée dans l'unité de radiobiologie du Centre d'Etude de l'Energie Nucléaire est, à travers des projets nationaux et internationaux, de fournir la connaissance scientifique nécessaire aux autorités et d'informer la population de façon adéquate quant aux effets des radiations dans les circonstances normales ou accidentelles.

Les principales lignes de recherches actuelles (également reprises dans la figure ci-dessous) de cette unité concernent:

* Le développement de biomarqueurs de la sensibilité individuelle aux radiations ionisantes et les risques de cancer y afférents.

* L'impact des radiations ionisantes sur les systèmes cardiovasculaire, cérébro-vasculaire, immunologique ainsi que sur le développement de l'embryon et du cerveau.

* L'impact des conditions spatiales (en particulier des radiations cosmiques et de l'apesanteur) sur la santé.

* Les effets biologiques de l'utilisation médicale des radiations ionisantes et des substances radioactives dans les domaines de la radiothérapie et de l'imagerie médicale.

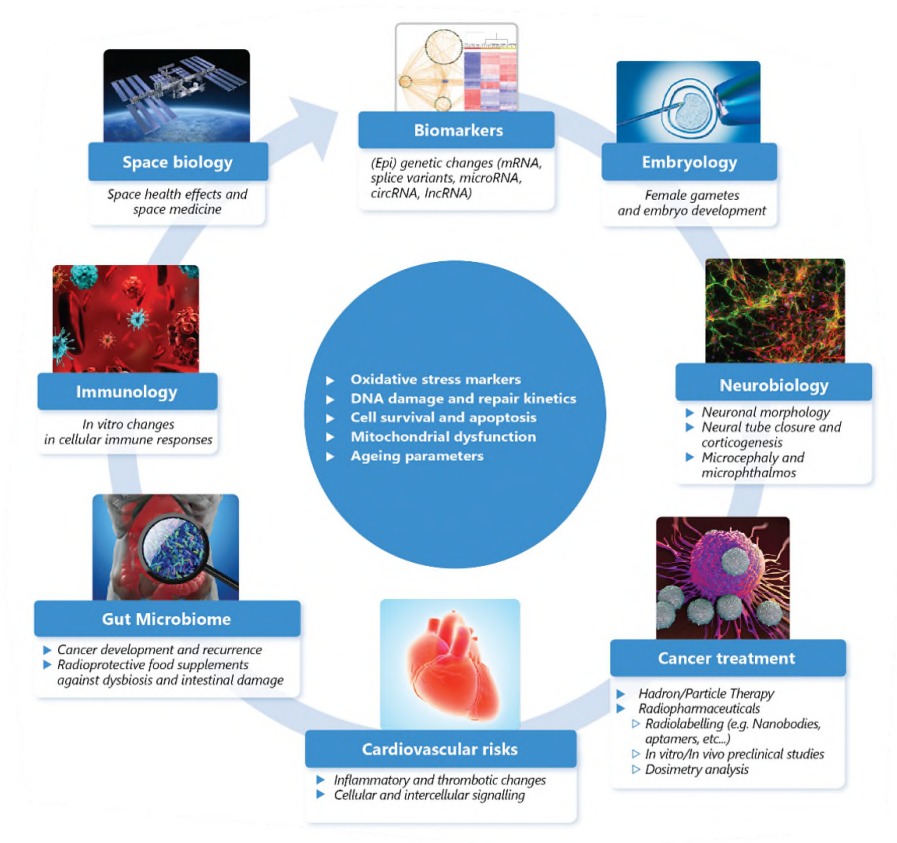




\section{Sensibilité individuelle aux radiations ionisantes et risques de cancer}

Cette recherche est réalisée pour :

* comprendre les mécanismes moléculaires à la base des effets des radiations.

* déterminer si les faibles doses de radiations peuvent induire des cancers et enfin.

* préciser dans quelle mesure certains facteurs génétiques peuvent moduler le risque de cancer aux faibles doses.

La recherche sur les marqueurs moléculaires est aussi utilisée actuellement à des fins diagnostiques (par exemple: patients pédiatriques passant des examens de tomographie assistée par ordinateur ou biosurveillance de la population en cas d'accident nucléaire).

\section{Effets des radiations ionisantes sur le développement de l'embryon et du cerveau}

Des patientes peuvent être exposées aux radiations ionisantes à des fins thérapeutiques pendant la grossesse lorsque la patiente nécessite un traitement radiothérapeutique contre un cancer agressif qui ne peut être entamé après la naissance de l'enfant. Les risques potentiels d'une telle exposition pour l'embryon dépendent partiellement de sa constitution génétique. Des souris possédant différents backgrounds génétiques sont utilisées comme modèles pour prédire les effets potentiels des radiations ionisantes en matière de mortalité prénatale, de malformations congénitales ou de retards de croissance, ainsi que les mécanismes moléculaires sous-jacents. Les effets cognitifs induits par des doses faibles ou modérées de radiation, tels que ceux observés parmi les survivants d'Hiroshima et Nagasaki, constituent un problème crucial en radioprotection. La radiosensibilité du cerveau au début de l'embryogenèse et les mécanismes du retard mental radio-induit sont étudiés par l'utilisation conjointe de modèles animaux, de la culture cellulaire in vitro et de techniques moléculaires.

\section{Hadronthérapie}

L'hadronthérapie, ou thérapie des particules, est une forme relativement nouvelle de radiothérapie externe, au cours de laquelle le patient est irradié avec des particules chargées (protons ou ions carbone, au lieu des rayons-X). Dans ce domaine, nous menons des recherches tant sur les aspects physiques que biologiques liés à la thérapie par le carbone et les protons. D'un côté, nous caractérisons la réponse de différents types de radiations en fonction de la dose, dans des champs de particules. D'autre part, nous étudions la réponse moléculaire aux ions carbone et aux protons de différents modèles cellulaires.

\section{Définition de la composante génétique du risque de cancer de la thyroüde aux faibles doses}

Le cancer de la thyroïde figure parmi les tumeurs malignes induites par une exposition aux radiations, ainsi que l'attestent les études épidémiologiques réalisées sur différentes populations exposées aux radiations telles que: les survivants des bombardements atomiques au Japon, les habitants des Iles Marshall exposés aux retombées des essais nucléaires et les enfants traités par radiothérapie au niveau de la tête ou de la nuque ou ceux exposés accidentellement aux radiations 
comme lors de l'accident de Tchernobyl. Cependant, on ne dispose pas encore de modèle adéquat pour caractériser la relation dose-effet aux faibles doses. C'est la raison pour laquelle nous étudions actuellement les facteurs génétiques susceptibles d'influencer le risque de développer un cancer de la thyroïde suite à une exposition à de faibles doses de radiations.

\section{Des molécules couplées à des radionucléides pour combattre le cancer: premières étapes du $S C K \bullet C E N$ dans le domaine de la radiothérapie ciblé}

$\mathrm{Au}$ cours des dernières décennies, il y a eu une demande croissante de nouveaux agents radiopharmaceutiques de la part du secteur médical. L'une des applications les plus importantes de ce type de molécules est la thérapie du cancer. Actuellement, nous étudions l'efficacité de différents agents radiopharmaceutiques.

\section{Recherche en biologie spatiale: comment l'espace peut affecter la santé des astronautes}

Durant un vol spatial, les astronautes sont exposés aux radiations cosmiques émanant du soleil et des galaxies. La dose reçue lors d'une mission dans la station spatiale internationale, en orbite à 300-400 km autour de la terre, peut être 100 à 200 fois supérieure à celle reçue sur terre durant une période équivalente, ce qui est loin d'être négligeable. Les effets biologiques des conditions spatiales sur l'homme sont fort complexes et encore mal connus. La continuation des recherches est donc essentielle en prévision des longs vols spatiaux. C'est pour cette raison que nous étudions les changements cellulaires, biochimiques, génétiques ou épigénétiques induits dans les cellules des astronautes, grâce à des technologies de haut débit. Au SCK•CEN, les radiations et la simulation de la microgravité sont également particulièrement étudiées in vitro. Nous avons d'autre part aussi recours à des plateformes terrestres analogues à celles de l'espace (stations en Antarctique Concordia et Princess Elisabeth, Bed Rest et Mars 500).

\section{Marie Sklodowska-Curie et le 7 novembre comme journée de la radiobiologie et de la médecine des radiations:}

Marie Skłodowska Curie (7 novembre 1867 - 4 juillet 1934) était physicienne et chimiste polonaise, naturalisée française ayant mené des recherches pionnières sur la radioactivité. Elle a été la première femme à remporter un prix Nobel, la première et unique femme à en remporter par deux fois, la seule à remporter un prix Nobel dans deux sciences différentes et faisait partie de l'héritage de cinq prix Nobel de la famille Curie. Elle a également été la première femme à devenir professeure à l'Université de Paris et, en 1995, elle est devenue la première femme à être enterrée au Panthéon à Paris.

Récipiendaire de prix Nobel de physique et de chimie, Marie Sklodowska-Curie a ouvert la voie à la physique nucléaire, à la chimie ainsi qu'à la radiobiologie et au traitement du cancer. Pour coïncider avec sa naissance, il est proposé que le 7 novembre devienne la Journée de la radiobiologie et de la médecine des rayonnements. Vous pouvez soutenir ce mouvement sur le site suivant : https://www.sckcen.be/DayOfRadiation 
Bulletin de la Société Royale des Sciences de Liège

Vol. 87, Actes de Colloques, La radioactivité, 2018, p. 1 - 7

\section{$\underline{\text { IV. Références }}$}

- Christensen DM, Iddins CJ, Sugarman SL, "Ionizing radiation injuries and illnesses". Emerg Med Clin North Am. 32 (1): 245-65, 2014.

- Committee to Assess Health Risks from Exposure to Low Levels of Ionizing Radiation, National Research Council, Health Risks from Exposure to Low Levels of Ionizing Radiation: BEIR VII - Phase 2, Washington, DC, National Academy Press, 2006

- Little et al. "Risks associated with low doses and low dose rates of ionizing radiations: Why Linearity May Be (Almost) the Best We Can Do" Radiology 2009;251:6-12.

- Marie Curie - Biography. Nobelprize.org. 4 July 1934. Retrieved 1 August 2012. 